\section{Focal Acral Hyperkeratosis}

A 12-year old boy, presented with skin colored raised lesions on dorsum of his hands for 2 years. There were multiple, 1 to 4-mm, skin-colored, firm, discrete, smooth surfaced papules, present on the dorsal aspect of both hands. Skin biopsy revealed hyperkeratosis, hypergranulosis, acanthosis and a normal dermis. Elastin stain did not reveal any degeneration. The child was diagnosed with focal acral hyperkeratosis. The differential diagnosis include verruca vulgaris, acrokeratoelastoidosis of Costa, degenerative collagenous plaques of the hands, keratoelastoidosis marginalis of the hands, punctate palmoplantar keratoderma, digital papular calcinosis, acral mucinosis, acrokeratosis verruciformis of Hopf and xanthoma. The skin lesions in hereditary papulotranslucent acrokeratoderma are characteristically exacerbated after exposure to water. Marginal papular acrokeratoderma encompasses a group of disorders that share crateriform, keratotic papules along the margins of the hands and feet. Warts exhibit verrucous surface as compared to smooth papules of focal acral hyperkeratosis. Punctate palmoplantar keratoderma is associated with thickened palms and soles, acral mucionosis and xanthoma have a shiny white coloured appearance. Probably a biopsy with special staining (presence of elastorrhexis, mucin, fat, calcium deposits) would aid in their definite differentiation.

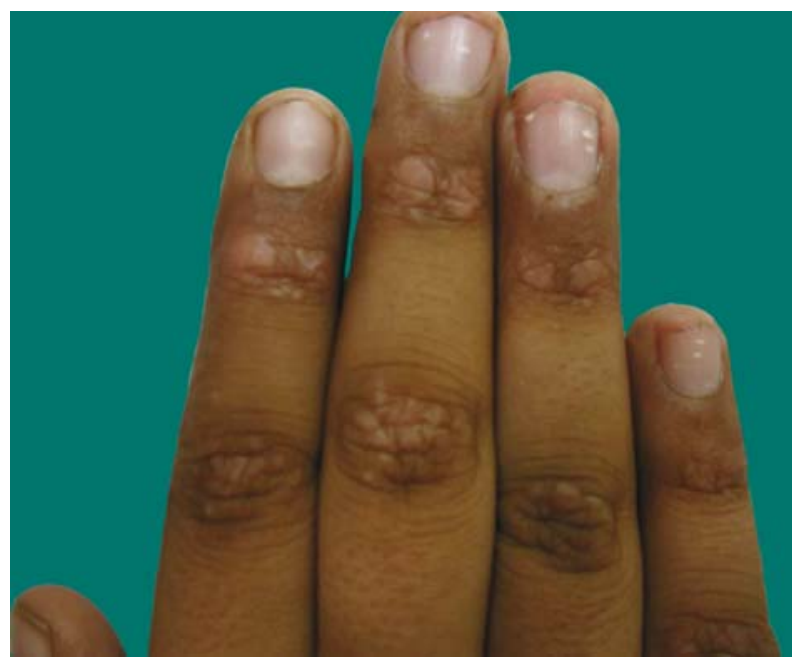

FIG.1 Multiple skin-colored papules.

Although the condition is benign, it may cause psychological and cosmetic concern to the patient. Multiple therapies have been attempted like topical keratolytics (urea, salicylic acid, and lactic acid), topical retinoids (tazarotene) and systemic agents like acitretin. Recurrences are reported on discontinuation.

Kabir Sardana, Shikha Chugh and ViJay K Garg Department of Dermatology, Maulana Azad Medical College and Lok Nayak Hospital, New Delhi, India. kabirijdvl@gmail.com

\title{
Heat Injury
}

Hypothermia is an important problem in neonates, especially in those with low birth weight. The most effective/safe and scientifically proven means of temperature maintenance at home is kangaroo mother care. Indigenous methods of temperature maintenance such as using hot water bags, warm cotton, heaters, blowers etc. could lead to burns and heat injury. A 5-dayold boy was brought to our hospital with vesiculo-bullous eruptions on both feet. The grandmother, after noticing that the baby was getting cold, kept the child very close to the fire for about 2 hours. The differential diagnose of such bullous lesions in a newborn are: staphylococcal scalded skin syndrome, epidermolysis bullosa, congenital erythropoeitic porphyria, herpes simplex and congenital syphilis. The treatment of such a patient includes supportive care, antibiotics and surgical drainage of pus.

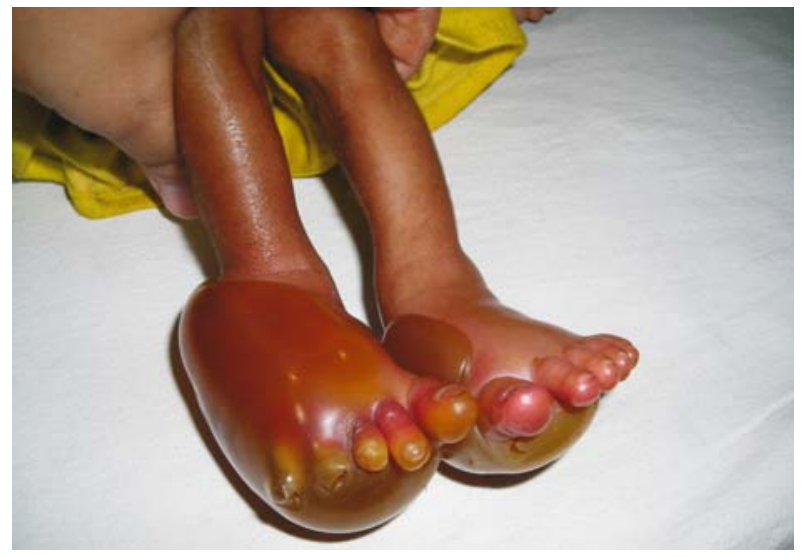

FIG.1 Bullous swelling over right foot.

RUCHI RAI, DK SINGH AND *JK SINGH, Departments of Pediatrics and *Surgery, MLN Medical College, Allahabad, Uttar Pradesh, India. ruchiraialld@gmail.com 